\title{
Velocity Field of the Planetary Nebula Wray 16-423
}

\author{
Krzysztof Gesicki \\ CA UMK, ul.Gagarina 11, Torun, Poland
}

Albert A. Zijlstra

UMIST, Department of Physics, P.O. Box 88, Manchester, UK.

Abstract. We have applied the "Torun models" to the PN Wray 16423 , and found evidence for an embedded shock in the nebula.

\section{The photo-ionization model}

The planetary nebula Wray $16-423$ is located at a distance of $25 \mathrm{kpc}$ in the Sagittarius Dwarf Galaxy. We obtained a 2-hour echelle spectrum at the ESO NTT telescope, with a resolution of 60000 covering 4000-9500 $\AA$. The resolved emission line profiles can be used to obtain a radial velocity profile. The best model parameters as well as the chemical composition are adopted from literature. For the analysis we selected nine strong and well exposed lines which cover a broad range of excitation potentials, six of them are shown in Fig. 1.

The observed nebular diameter is about 1 arcsec. The high excitation lines (HeII, [ArIV], [OIII]) from the high density inner region do not show line splitting, in agreement with the observed diameter. However, lower-excitation lines show some line splitting, and must form in a region slightly larger than 1 arcsec, in a lower density halo. We adopt an outer radius of $0.09 \mathrm{pc}$, and a density structure for which the calculated surface brightness in $\mathrm{H} \beta$ decreases to $10 \%$ of maximum at the diameter of 1 arcsec. This density is represented by dotted line in right panel of Fig. 1. No high-resolution image is available to confirm this deduced structure.

\section{Velocity and density fields}

Velocity fields of PNe commonly show low-velocity gas near the inner edge, with velocity increasing approximately as $r^{2}$. This fails in Wray 16-423, because the HeII line is too broad: it requires a high velocity $(50 \mathrm{~km} / \mathrm{s})$, high density region near the inner nebular radius. Further out the velocity must decrease significantly (to about $15 \mathrm{~km} / \mathrm{s}$ ) to reproduce the narrow cores of the [OIII] and [ArIV] lines. The acceleration to $60 \mathrm{~km} / \mathrm{s}$ in the outermost layers, seen in the extended wings of the low-excitation lines, is a common feature of PNe.

This three-component velocity field (dashed line in Fig. 1) reproduces relatively well the general shape of all lines, however it is clearly visible that a component expanding about $40 \mathrm{~km} / \mathrm{s}$ is missing. 

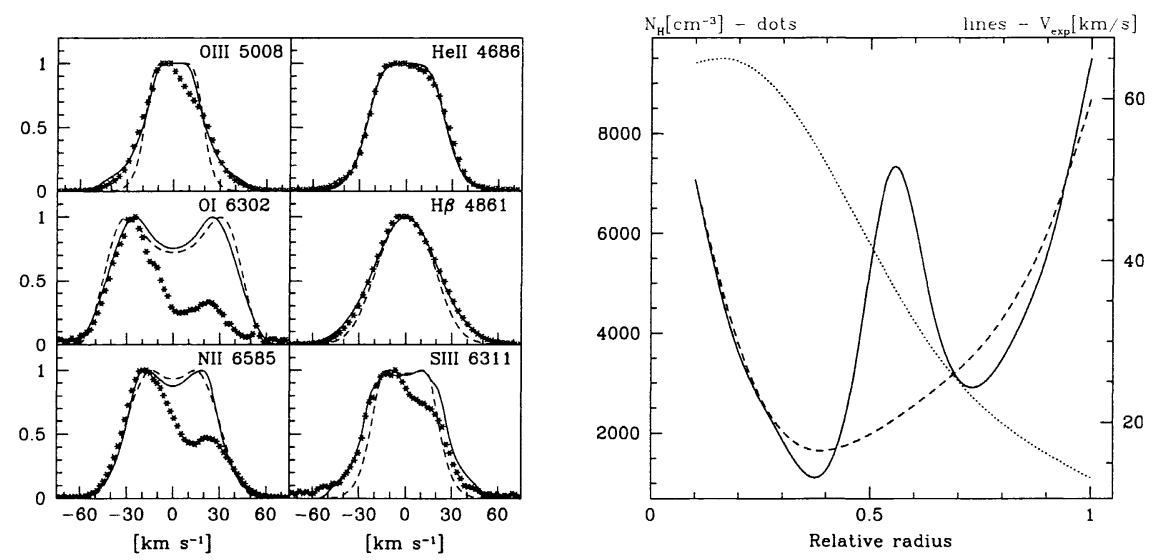

Figure 1. Left panel: fitted lines with a smooth velocity field. The points represent the observed spectrum, and the lines show the modelled profiles. Right panel: model parameters. The dotted line gives the density distribution, and the solid lines show two different velocity distributions.

The velocity profile of the best model is shown in Fig. 1 as continuous line. It is highly peculiar, and gives the impression of an embedded shock. If this is correct, the density should also be enhanced in this region which would make the high velocity region even thinner. We cannot be certain that this represents a shock, but the model is highly suggestive.

\section{Discussion}

The density and velocity enhancement at the inner radius suggest that Wray16423 has developed a strong stellar wind from the central star. This behavior can be expected for the very hot star. This is also consisted with the wind features: the star is classified as 'wels' (weak-emission line star). The acceleration at the outer radius results from the ionization front. But the embedded shock remains unexplained. Possibly, the fast wind has broken through the (clumpy?) dense shell and set up a second wind-swept shell.

Why is our rather old PN ionization bounded? Was the superwind denser and removed more mass than usually assumed? Or is Wray 16-423 in a phase of recombination, when the central star starts to decrease in brightness and there are not enough photons to ionize the whole superwind? To answer these question requires hydrodynamical calculations.

Acknowledgments. This work was supported by Polish KBN grant No. 2.P03D.020.17 